Max-Planck-Institut für demografische Forschung Max Planck Institute for Demographic Research Konrad-Zuse-Strasse $1 \cdot$ D-18057 Rostock · GERMANY

Tel +49 (0) 3812081 - 0; Fax +49 (0) 3812081 - 202;

http://www.demogr.mpg.de

MPIDR WORKING PAPER WP 2003-039

DECEMBER 2003

\title{
Age Correspondence \\ for Different Mortality Regimes with and without the Change Point
}

M.S. Finkelstein (FinkelM@sci.uovs.ac.za)

This working paper has been approved for release by: James W. Vaupel (jwv@ demogr.mpg.de)

Head of the Laboratory of Survival and Longevity.

(C) Copyright is held by the authors.

Working papers of the Max Planck Institute for Demographic Research receive only limited review. Views or opinions expressed in working papers are attributable to the authors and do not necessarily reflect those of the Institute. 


\title{
AGE CORRESPONDENCE \\ FOR DIFFERENT MORTALITY REGIMES WITH AND WITHOUT THE CHANGE POINT
}

\author{
M.S. Finkelstein \\ Department of Mathematical Statistics \\ University of the Free State \\ PO Box 339, 9300 Bloemfontein, Republic of South Africa \\ (e-mail: FinkelM@sci.uovs.ac.za) \\ and \\ The Max Planck Institute for Demographic Research, \\ Rostock, Germany
}

Draft: 6 October 2003

\begin{abstract}
The mortality rates are steadily declining with time. The remaining lifetime for e.g. 65 years old person even 20-30 years ago was substantially smaller than nowadays. Therefore, the age correspondence problem for populations in different mortality regimes is of interest. A simple solution, based on the equality of accumulated mortality rates (or, equivalently, on the equality of probabilities of survival) is considered. Furthermore, the mortality regime with a change point is defined and the procedure of age re-calculation after the change point is suggested. Two age recalculation models (and their combination) are discussed: the first one accounts for wear accumulation in the process of aging and the other is characterized by a kind of memoryless property.
\end{abstract}

Keywords: Life span; Mortality regime; Change point; Age re-calculation. Sedjakin Principle.

\section{INTRODUCTION. AGE CORRESPONDENCE.}

Is sixty-five, which was regarded in developed countries for a long time as the threshold for the definition of the entry into the "old age", really a marker of the old age nowadays? Given the steady decline in mortality rates for a number of decades, the answer will be definitely negative. In order to define this marker age properly it is reasonable to look at are more general problem of "recalculating" the age of populations at different times.

Let, as usually, $\mu(x, t)$ denote the force of mortality (mortality rate) as a function of age $x$ and time $t$. Let $t=t_{b}$ be some reference (baseline) time, which defines the baseline mortality regime. We shall consider the "real" or current regime defined usually at a later point in time: $t=t_{r}>t_{b}$. Without mentioning it further, we shall deal with an adult mortality when mortality rates are increasing in $x$. For simplicity of notation, however, assume also that the support of the corresponding lifetime (age at death) distribution (Cdf) $F(x, t)$ is $R:[0, \infty)$ ).

It is well-known that the demographic data shows the decrease in mortality rates in time:

$$
\mu_{b}(x) \equiv \mu\left(x, t_{b}\right) \geq \mu\left(x, t_{r}\right) \equiv \mu_{r}(x) ; t_{r} \geq t_{b}, \forall x \in[0, \infty)
$$


which leads to the following stochastic ordering (Shaked and Shantikhumar (1993)) of the corresponding Cdfs:

$$
F_{b}(x) \geq F_{r}(x) ; \forall x \in[0, \infty)
$$

and therefore, to a weaker property of increasing life expectancy (as well as the remaining life expectancy) in $t$.

The specific case of ordering (1) is the Gompertz mortality change model (Bongaarts and Feeney, 2002) with a constant shift in $x$ on a logarithmic scale for each fixed $t$ :

$$
\mu(x, t)=\mu(0, t) \exp \{b x\}
$$

where $b>0$ is a parameter. In the concrete example of this paper the corresponding regimes were set by $t_{b}=1951 ; t_{r}=1995$ for US females.

Most of conventional approaches to defining in a comparative way the age of entry into the old age for different regimes are based on the concept of remaining lifetime. Thus, the commonly used criterion (e.g. Siegal, 1993) is given by the value $x_{r}$ obtained from the equation for remaining life expectancies:

$$
\frac{\int_{x_{b}}^{\infty} l\left(x, t_{b}\right) d x}{l\left(x_{b}, t_{b}\right)}=\frac{\int_{x_{r}}^{\infty} l\left(x, t_{r}\right) d x}{l\left(x_{r}, t_{r}\right)}
$$

where $x_{b}$ is the definition of the old age in a baseline regime (e.g., 65 years) and $l\left(x, t_{b}\right), l\left(x, t_{r}\right)$ are the life table probabilities (proportions of persons surviving to age $x$ ) for the baseline and real regimes respectively:

$$
l(x, t)=\exp \left\{-\int_{0}^{x} \mu(u, t)\right\} d u
$$

It is clear that equation (3) can be used as some age correspondence equation for 'arbitrary' ages $x_{r}$ and $x_{b}$ and not only for the purpose of defining the old age. This correspondence might be reasonable in actuarial applications for defining and comparing retirement ages for different regimes, for instance. However, it is clear that condition (3) definitely increases the proportion of old people in the less severe regime at time $t_{r}$, as compared with the regime at $t_{b}$.

On the other hand, the natural and very simple (maybe oversimplified) age correspondence rule for two regimes is to find $x_{r}$ for each $x_{b} \geq 0$ from the following equation:

$$
\int_{0}^{x_{b}} \mu_{b}(x) d x=\int_{0}^{x_{r}} \mu_{r}(x) d x .
$$

Example 1. Let $\mu(0, t)$ in relation (2) for simplicity be given as

$$
\mu(0, t)=\exp \{-\alpha t\} ; \alpha>0 \text {. }
$$

By direct integration in (5):

$$
x_{r}=\frac{\ln \left\{\left[\exp \left\{\alpha\left(t_{r}-t_{b}\right\}\left(\exp \left\{b x_{b}\right\}-1\right)\right]+1\right\}\right.}{b}>x_{b} .
$$

Performing under obvious assumptions the expansion in the Taylor's series:

$$
x_{r} \approx x_{b}\left[1+\alpha\left(t_{r}-t_{b}\right)\right]
$$

we arrive at the reasonable approximate relation. 
Due to exponential representation (4), equation (5) leads to equality of the corresponding life table probabilities

$$
l\left(x_{b}, t_{b}\right\}=l\left(x_{r}\left(x_{b}\right), t_{r}\right)
$$

which means that the condition, stating equal probabilities of survival for different mortality regimes is the basis for obtaining the re-calculation rule: $x_{r}$ as a function of $x_{b}: x_{r}\left(x_{b}\right)$.

Remark 1. The described procedure and, specifically, equation (6) defines, in fact, the 'life table analogue' of the accelerated life model (Finkelstein (1998), Cox and Oakes (1984)). Indeed, using the conventional for survival analysis notation for equation (4), we can write for the baseline regime $(\bar{F} \equiv 1-F)$ :

$$
l\left(x, t_{b}\right\}=\bar{F}_{b}(x),
$$

whereas for the lighter regime at $t=t_{r}$ :

$$
l\left(x, t_{r}\right)=\bar{F}_{r}(x)=\bar{F}_{b}(W(x)),
$$

which for monotone increasing $W(x)$ defines the general ALM. The specific linear model: $W(x)=W x$, where $W>0$ is widely used in applications. Using exponential representations for $\bar{F}_{r}(t)$ and $\bar{F}_{b}(x)$, the scale transformation function $W(x)$ can be easily obtained from the equation similar to (5):

$$
\int_{0}^{x} \mu_{r}(u) d u=\int_{0}^{W(x)} \mu_{b}(u) d u
$$

Comparing relations (5) and (7):

$$
x=x_{r} ; x_{b}=W(x)
$$

and eventually:

$$
x_{r} \equiv x_{r}\left(x_{b}\right)=W^{-1}\left(x_{b}\right),
$$

where $W^{-1}(x)$ denotes the inverse function to $W(x)$, which is clearly also increasing. It is also clear, that as $t_{r}$ defines the lighter regime: $W^{-1}(x) \geq x ; x \geq 0$, whereas $W(x) \leq x ; x \geq 0$.

Denote $W^{-1}(x) \equiv \tilde{W}(x)$. In what follows it is more convenient to use the function $\tilde{W}(x)$ rather than $W(x)$. The age correspondence equation (8) can be equivalently written as

$$
\int_{0}^{x} \mu_{b}(u) d u=\int_{0}^{\tilde{W}(x)} \mu_{r}(u) d u .
$$

Using definition of the force of mortality the following important relationship between the failure rates in two regimes can be obtained from equation (7)(Alternatively via differentiation with respect to $x$ of both sides of equation (8)):

$$
\mu_{r}(x)=W^{\prime}(x) \mu_{b}(W(x))
$$

Remark 2. The suggested approach to defining the age correspondence for populations at different times is rather general and is based on the assumption of an 'invariant nature' of the functional of the accumulated mortality rate 


$$
M(x, t)=\int_{0}^{x} \mu(u, t) d u
$$

The importance of this functional in age correspondence problems was first indicated by Sedajakin (1966) and later was discussed and used by many authors (see, e.g., Nelson (1990), Liu and Makish (1996)) in the reliability-oriented publications. The main assertion, which is known in Russian reliability literature as the Sedjakin Principle, states:

If the functioning regime of an object had been changed at some point of time $x_{0}$, its further trajectory of performance in $\left[x_{0}, \infty\right)$ does not depend on the whole history of its performance in $\left[0, x_{0}\right)$, but only on the value of accumulated rate $M\left(x_{0}, t\right)$.

This is a specific, simple way of using the aggregated information on the history of performance in stochastic modeling of the future performance of an object. There is a lot of evidence in the literature on accelerated testing (Nelson (1990)) that this principle holds for rather mild reasonable assumptions. We shall use it in the next section.

Remark 3. Speaking strictly, the suggested age correspondence is working without distortions only for the model case of closed stationary populations. The influence of history of fertility and migration on the model should be investigated as a special topic.

Remark 4. Using period tables in practice means that mortality rates are overestimated from a cohort point of view, as $\mu(x, t)$ is declining in $t$. However the rates are overestimated for both $t_{b}$ and $t_{r}$ which provides an offset to a certain extent (Vaupel (2002), Denton and Spencer (1997)).

We end this section with the following interpretation of the age-recalculation (with respect to life expectancy) results of Oeppen and Vaupel (2002). An astonishing fact was stated in this paper: Female life expectancy in the record holding country (for the last 40 years this country is Japan) has risen linearly with almost 3 months increase per year. This linear pattern suggests that the phenomenon can be interpreted (modeled) via the linear ALM. Indeed, let $X_{t_{b}}$ and $X_{t}$ be the population lifetime random variables at $t_{b}$ and $t \geq t$, respectively. Let $k>0$ be a constant. Assume that:

$$
X_{t}=\left(1+k\left(t-t_{b}\right)\right) X_{t_{b}}
$$

which obviously define the specific linear case of ALM (7). Applying operation of mathematical expectation to both sides of equation (12) results in the linear model of Oeppen and Vaupel (2002)

$$
E\left[X_{t}\right]=\left(1+k\left(t-t_{b}\right)\right) E\left[X_{t_{b}}\right]=\left(1-k t_{b}\right) E\left[X_{t_{b}}\right]+k t E\left[X_{t_{b}}\right],
$$

where for the specific case considered in this paper: $t_{b}=1960, t \in[1960,200]$ and the corresponding slope is $\left(1-k t_{b}\right) E\left[X_{t_{b}}\right]=0.243$. It is clear that the ALM assumption (12) is stronger than the linear representation for life expectancy. Verification of relation (12) for this specific case presents an interesting problem, although the available data could be insufficient for this task. It is worth mentioning that the general form of (12) for the situation under consideration can be written as 


$$
X_{t}=\left(1+\hat{W}\left(\left(t-t_{b}\right), x\right) X_{t_{b}},\right.
$$

where $\hat{W}\left(\left(t-t_{b}\right), x\right)$ is a function of time and age. The reasonable decomposition of this function could be $\hat{W}\left(\left(t-t_{b}\right), x\right)=f\left(t-t_{b}\right) g(x)$. Model (12) is its specific, when the function $f(t)$ is linear and $g(x) \equiv 1$.

\section{A MODEL OF MORTALITY WITH REGIME CHANGE POINT.}

The decline in the force of mortality with $t$ shows some general pattern of human mortality in time. On the other hand, the influence of environment (by environment we mean some general external characteristics describing external risks, quality of life, lifestyle, etc) on population for the fixed $t$ is also of interest.

The most popular in survival analysis and reliability way to deal with the influence of environment is to describe it via the proportional hazards $(\mathrm{PH})$ model:

$$
\mu_{r}(x)=z \mu_{b}(x)
$$

where $z>0$ is a parameter which describes the multiplicative impact of environment on the baseline hazard (mortality rate) $\mu_{b}(x)$, and $\mu_{r}(x)$ is the mortality rate in the 'real' (under investigation) environment. In regression models usually $z=\exp \left\{x^{T} y\right\}$, where $x=\left(x_{1}, \ldots, x_{n}\right)$ is a vector of time-independent covariates and $y=\left(y_{1}, \ldots, y_{2}\right)$ is a vector of unknown parameters. Thus, environment can be characterized by a large number of different covariates and it is important for analysis to define those, which are really important. In a more general form (13) can be written with a time-dependent $z(x)$.

Consider a population in some reference (baseline) environment (regime) with the corresponding mortality rate $\mu_{b}(x)$. Denote by $z_{b}(x)$ the regime, which formally corresponds to the baseline mortality rate: $\mu_{r}\left(x, z_{b}(x)\right) \equiv \mu_{b}(x)$, whereas the mortality rate in the arbitrary (real) regime $z(x)$ is denoted by $\mu_{r}(x, z(x))$. Assume that the initial baseline regime $z_{b}(x)$ is switched to a lighter one $z(x)$ at $x=x_{0}$. There can be numerous reasons for this model: e.g., radiation or pollution elimination in some area, change in the lifestyle, etc. Denote by $z_{c}(x)$ the resulting compound regime

$$
z_{c}(x)= \begin{cases}z_{b}(x), & 0 \leq x<x_{0} \\ z(x), & x_{0} \leq x\end{cases}
$$

In accordance with the Sedyakin Principle of the previous section (see equations (9) and (10)) the starting re-calculated population age in regime $z(x)$ (it can be also called equivalent or virtual age (Finkelstein (1997))) at $x=x_{0}$ is defined by $\tilde{W}\left(x_{0}\right)>x_{0}$. Therefore, the Cdf of age (at death) for the compound regime $z_{c}(x)$ is:

$$
F_{c}(x)= \begin{cases}1-\exp \left\{-\int_{0}^{x} \mu_{b}(u) d u\right\}, & 0 \leq x<x_{0} \\ 1-\exp \left\{-\int_{0}^{x} \mu_{r}\left(u+\tilde{W}\left(x_{0}\right), z\left(u+\tilde{W}\left(x_{0}\right)\right) d u\right\},\right. & x \geq x_{0}\end{cases}
$$

It is clear that the second line in the right hand side of (15) can be transformed to 


$$
\exp \left\{-\int_{\tilde{W}\left(x_{0}\right)}^{x+\tilde{W}\left(x_{0}\right)} \mu_{r}(u, z(u) d u\} .\right.
$$

Denote for brevity: $\mu_{r}(x) \equiv \mu_{r}(x, z(x))$. In accordance with (15), the survival function of the remaining lifetime after the switch at $x_{0}$ is

$$
\bar{F}_{c}\left(x \mid x_{0}\right)=\frac{\bar{F}_{c}\left(x_{0}+x\right)}{\bar{F}_{c}\left(x_{0}\right)}=\exp \left\{-\int_{x_{0}+\tilde{W}\left(x_{0}\right)}^{x_{0}+\tilde{W}\left(x_{0}\right)+x} \mu_{r}(u) d u\right\} .
$$

On the other hand, this function without the switch would be:

$$
\bar{F}_{b}\left(x \mid x_{0}\right)=\frac{\bar{F}_{b}\left(x_{0}+x\right)}{\bar{F}_{b}\left(x_{0}\right)}=\exp \left\{-\int_{x_{0}}^{x_{0}+x} \mu_{b}(u) d u\right\}
$$

It was assumed that $z(x)$ is a lighter regime than the baseline $z_{b}(x)$. This notion should be specified. Let mortality rates under consideration be monotonically increasing in $x$, which is a usual assumption for adult mortality.

Definition 1. The mortality regime $z(u)$ is lighter than the baseline mortality regime $z_{b}(x)$ in a weak sense, if the following mortality rates ordering holds:

$$
\mu_{r}(x) \geq \mu_{b}(x) ; \forall x \in[0, \infty)
$$

This is a natural definition for comparing two different regimes without a change point. Let $F_{r}(x), x \geq 0$ denote the Cdf defined by the mortality rate $\mu_{r}(t)$. It is clear that in this case the following ordering for the remaining lifetimes:

$$
F_{r}\left(x \mid x_{0}\right) \leq F_{b}\left(x \mid x_{0}\right), \forall x, x_{0} \geq 0
$$

holds, which trivially leads to the corresponding ordering of life expectancies at $x_{0}$ as well. On the other hand, it is clear (see Example 2) that condition (18) does not guarantee that

$$
\mu_{r}\left(\tilde{W}\left(x_{0}\right)<\mu_{b}\left(x_{0}\right)\right.
$$

and, therefore, the mortality ordering at least for some $x^{\prime} \geq 0$ :

$$
\mu_{r}\left(\tilde{W}\left(x_{0}+x\right)<\mu_{b}\left(x_{0}+x\right), x \in\left[0, x^{\prime}\right),\right.
$$

is also not guaranteed, whereas it is natural to expect the opposite sign in inequalities (20) and (21) upon switching to the lighter regime. Thus, the stronger definition, for a lighter regime should be given, but, firstly, consider the following example:

Example 2: Let $\mu_{b}(x)=a x$ be linear and let $\mu_{r}(x)=a_{0}$ in $\left[0, x_{1}\right)$ and then be again linear with a slope $a_{1}>a$. For simplicity we can assume that $a_{0}=0$. Furthermore, let for $x \geq x_{2}=\left\{\left(a_{1}-a_{2}\right) / a_{1}\right\}$ the slope of $\mu_{r}(x)$ is the same as for $\mu_{b}(x): \mu_{r}(x)=a x$. It is clear from equation (10) that in this case (for the not very large $x_{0}$ ):

and inequality (20) does not hold.

$$
\mu_{r}\left(\tilde{W}\left(x_{0}\right)=\sqrt{a a_{1}} x_{0}>a x_{0}=\mu_{b}\left(x_{0}\right)\right.
$$

Definition 2. Let mortality rates ordering (18) holds for increasing mortality rates. Let the regime be switched at $x=x_{0}$, as defined by relation (14) and the starting age in 
regime $z(x)$ is $\tilde{x} \geq x_{0}$. (We do not assume the concrete rule (e.g., $\tilde{x}=\tilde{W}\left(x_{0}\right)$ ) of time re-calculation in this definition). Let:

$$
\left.\mu_{b}\left(x_{0}+x\right)-\mu_{r}(\tilde{x}+x)\right) \geq 0 ; \forall x_{0} \geq 0, x \geq 0 .
$$

Then the mortality regime $z(u)$ is defined as a lighter than a baseline one in a strong sense for an age re-calculation model with a regime change point at $x=x_{0}$.

Example 3. Let: $\mu_{b}(x)=a \exp \{x\}, \mu_{r}(x)=b \exp \{x\} ; a>b$. Thus, in accordance with Definition 1 , the mortality regime $z(u)$ is lighter than the baseline one in a weak sense. For applying Definition 2 consider:

$$
\left.\mu_{b}\left(x_{0}+x\right)-\mu_{r}(\tilde{x}+x)\right)=\exp \{x\}\left[a \exp \left\{x_{0}\right\}-b \exp \{\tilde{x}\}\right]
$$

It can be easily seen that this relation is positive (non-negative) when

$$
x_{0} \leq \tilde{x} \leq x_{0}-\ln (b / a),
$$

which means that the starting point after the re-calculation $\tilde{x}$ should not be too large to guarantee that the rate $\mu_{r}(\tilde{x}+x)$ is 'lower' than the baseline rate $\mu_{b}\left(x_{0}+x\right)$.

The similar reasoning holds e.g., for $\mu_{b}(x)=\exp \{a x\}, \mu_{r}(x)=\exp \{b x\} ; a>b$ or for the power law for the mortality rate function.

The following important and simple result is a straightforward consequence of Definition 2:

Theorem. Let the real regime be lighter than the baseline in the strong sense $\left(\tilde{x}=\tilde{W}\left(x_{0}\right)\right.$ ). Then the remaining lifetime, defined by the Cdf (16) is stochastically larger than the remaining lifetime, defined by the Cdf (17):

$$
\exp \left\{-\int_{x_{0}+\tilde{W}\left(x_{0}\right)}^{x_{0}+x+\tilde{W}\left(x_{0}\right)} \mu_{r}(u) d u\right\} \geq \exp \left\{-\int_{x_{0}}^{x_{0}+x} \mu_{b}(u) d u\right\} ; \forall x \geq 0
$$

The proof easily follows from the fact that $\tilde{W}\left(x_{0}\right)$ is the specific case of $\tilde{x}$ and equations (16), (17) and (22).

Remark 5. As it was mentioned before, the re-calculation condition (10) and inequality $\mu_{b}(x) \geq \mu_{r}(x) ;, \forall x \in[0, \infty)$ does not guarantee inequality (20) (see Example 2). It can be easily seen, however, that it holds e.g., for the setting of Example 3: $\mu_{b}(x)=a \exp \{x\}, \mu_{r}(x)=b \exp \{x\} ; a>b$. Applying the specific re-calculation rule (10) results in: $\tilde{W}\left(x_{0}\right)=\ln \frac{a\left[\exp \left\{x_{0}\right\}-1\right]+b}{b}$ and eventually:

$$
\mu_{r}\left(\tilde{W}\left(x_{0}\right)=a\left[\exp \left\{x_{0}\right\}-1\right]+b<a \exp \left\{x_{0}\right\}=\mu_{b}\left(x_{0}\right) .\right.
$$

Thus, relation (22) is also positive, which means that the real regime is lighter than the baseline one in a strong sense.

Differentiating (10) (where $x=x_{0}$ ) with respect to $x_{0}$ leads to a similar to equation (11) relationship between the mortality rates in different regimes:

It follows from (25) that if

$$
\mu_{b}\left(x_{0}\right)=\tilde{W}^{\prime}\left(x_{0}\right) \mu_{r}\left(\tilde{W}\left(x_{0}\right) .\right.
$$




$$
\tilde{W}^{\prime}\left(x_{0}\right)<1,
$$

condition (20) does not hold and the corresponding regime cannot be qualified as a lighter one in a strong sense. Thus inequality (26) presents a simple initial test for this property.

Example 3 (continued). It is clearly seen that (26) holds, as in this case:

$$
\tilde{W}^{\prime}\left(x_{0}\right)=\frac{a \exp \left\{x_{0}\right\}}{a\left[\exp \left\{x_{0}\right\}-1\right]+b}>1 \text {. }
$$

On the other hand, the mortality rates ordering (22) should hold for all values of $x \geq 0$ and not only for $x=0$ as in (25)-(26). It is clear that due to relation (23) in this specific case ordering (22) holds for all $x \geq 0$ as well.

It is worth mentioning that, as the life expectancy at some age $x_{0}$ for an age at death Cdf with mortality rate $\mu(t)$ is defined by:

$$
e\left(x_{0}\right)=\int_{0}^{\infty} \exp \left\{-\int_{\widetilde{x}}^{x+x_{0}} \mu(u) d u\right\} d x
$$

the corresponding inequality for life expectancies at $x_{0}$ (with and without the change point, respectively) trivially follows under assumptions of the Theorem:

$$
\int_{0}^{\infty} \exp \left\{-\int_{x_{0}}^{x+x_{0}} \mu_{b}(u) d u\right\} d x<\int_{0}^{\infty} \exp \left\{-\int_{x_{0}+\tilde{W}\left(x_{0}\right)}^{x_{0}+x+\tilde{W}\left(x_{0}\right)} \mu_{r}(u) d u\right\} d x .
$$

Therefore, the switch to the lighter in a strong sense regime increases the life expectancy.

\section{ACCUMULATION AND PLASTICITY}

The PH model (13) is a rather simple and widely used way of modelling an impact of environment on the baseline mortality rate. Let $z<1$, which means that $\mu_{r}(x)$ defines a lighter in a weak sense mortality regime. The PH model was discussed in the previous section only as one of the specific methods of ordering the mortality curves. On the other hand, similar to the accelerated life model (ALM), it can also define the alternative way of age re-calculation for the mortality regime with a change point. The corresponding rule can be interpreted as: no change in age. Similar to relation (15) the lifetime Cdf for the compound regime (14) is defined in this case as

$$
F_{c}(x)= \begin{cases}1-\exp \left\{-\int_{0}^{x} \mu_{b}(u) d u\right\}, & 0 \leq x<x_{0} \\ 1-\exp \left\{-\int_{0}^{x} \mu_{r}(u) d u\right\}, & x \geq x_{0}\end{cases}
$$

which means that age re-calculation function is zero: $\tilde{W}\left(x_{0}\right)=0$. In accordance with (27), the survival function of the remaining lifetime after the switch at $x_{0}$ is

$$
\bar{F}_{R}\left(x \mid x_{0}\right)==\exp \left\{-\int_{x_{0}}^{x_{0}+x} \mu_{r}(u) d u\right\}
$$


The re-calculation rule defined by relations (27) and (28) means that the population mortality history (before $x_{0}$ ) does not contribute to the mortality future (a kind of simplified Markovian property). Alternatively, the re-calculation procedure of the previous section, based on the ALM, takes into account accumulated wear in $\left[0, x_{0}\right)$ defined by the corresponding functional (12) in this interval of time.

What model (or combination of models) explains better the patterns of a 'real life mortality' with a change point? The survival analysis literature on testing the data on PH or ALM models is quite intensive, and the corresponding approaches can be used, in principle, for demographic purposes as well. But firstly, and this is very important, there should be at least some preliminary analysis of the corresponding aging processes. If, due to some reason, there is an evidence of the memoryless property, then model (27)-(28) can present a suitable tool for analysis. Latest experiments on the diet constraints for the fruit flies and other species support this idea (see Vaupel et al (2003), Mair et al, and Kolata (2003)). If, on the other hand, it is clear that the wear accumulation process governs aging, then the ALM and the Sedjakin Principle are more likely to account for this.

A useful for interpretation of the PH model can be found in the following shockbased setting. Assume now that the only source of an object's failure (death) is an environment, which is modeled by the Poisson point process of potentially harmful events-shocks with rate $\lambda_{s}(t)$. Each shock independently of previously survived shocks leads to failure with probability $\theta(t)$, which, e.g., shows an ability of an organism to survive the shock (repair capacity in Vaupel and Yashin (1987)). Thus, the object does not experience wear-out in the traditional sense. Then the well known result, which follows from Block et al (1985) or Finkelstein (2000) states that the corresponding lifetime survival function is defined by

$$
\bar{F}(x)=\exp \left\{-\int_{0}^{x} \theta(u) \lambda_{s}(u) d u\right\}
$$

Thus, the function

$$
\mu(x)=\theta(x) \lambda_{s}(x)
$$

is the mortality rate, defined by the Cdf (29). Applying this formula to the baseline regime $z_{b}(x)$ (baseline Poisson shock process with rate $\lambda_{s b}(t)$ and the baseline probability $\left.\theta_{b}(x)\right)$ and to a lighter regime $z(x)$ :

$$
\lambda_{s r}(x)<\lambda_{s b}(x) ; x \geq 0, \theta_{r}(x)<\theta_{b}(x) ; x \geq 0
$$

it is easy to obtain the following expression

$$
\mu_{r}(x)=\theta_{r}(t) \lambda_{s r}(x)=\theta_{b}(x) \lambda_{s b}(x) \frac{\theta_{r}(x) \lambda_{s r}(x)}{\theta_{b}(x) \lambda_{s b}(x)}=g(x) \mu_{b}(x)
$$

where $g(x)<1$ characterizes a lighter mortality regime, and there is no need in this case two define two types of a lighter regime, as in the previous section. Relation (31) defines a time-dependent version of the PH model (13).

Thus the effect of the change in environment in the PH model results in immediate change in the mortality rate in accordance with relations (13) or (31). The age of the object immediately after the change point is the same as it was just prior it (compare with $x_{0}$ and $\tilde{W}\left(x_{0}\right)$, respectively for the model defined by equations (19) and (20)). This memoryless model was called in Finkelstein (1998) "the absolute elasticity model", but the term "plasticity" is a more appropriate one (see Vaupel et al (2003) and Scholtz and Mair (2003)). 
Remark 6. It follows from results of this and the previous sections that after switching to a weaker regime the mortality rate defined via the Sedjakin Principle is larger than the one obtained via the PH model (for the same initial setting, of course):

$$
\mu_{r}\left(x_{0}+x\right)<\mu_{r}\left(\tilde{W}\left(x_{0}\right)+x\right), x \geq 0 .
$$

\section{COMBINED MODEL}

It is reasonable to assume that the 'real life model' can be described by the combination of these two models. By this we mean that the change in environment affects the part of an object (which is somehow accounts for wear accumulation or aging in a traditional sense) in accordance with the ALM and Sedjakin Principle. On the other hand, the complement part of an object is affected in accordance with the PH model ("elastic" part). Under the natural simplifying assumption of statistical independence of both parts (for the random environment, for instance, this assumption does not hold and the corresponding bivariate models should be considered), the survival probability is defined by the product rule:

$$
\bar{F}(x)=\bar{F}_{P H}(x) \bar{F}_{A L M}(x)
$$

or via the corresponding mortality rates:

$$
\mu(x)=\mu_{P H}(x)+\mu_{A L M}(x)
$$

Remark 7. It is also reasonable to assume that there is another 'stable' part of an organism, which is not influenced by changes in environment. In this case relation (32) turns to:

$$
\bar{F}(x)=\bar{F}_{s t}(x) \bar{F}_{P H}(x) \bar{F}_{A L M}(x),
$$

where $\bar{F}_{s t}(x)$ denotes the survival probability of this part. The forthcoming relations can be easily adjusted to this case.

Consider for the model (32)-(33), as previously, the baseline and the lighter regimes, respectively (for applying the Sedjakin Principle we must assume the lighter in a strong sense mortality regime):

$$
\mu_{b}(x)=\mu_{b P H}(x)+\mu_{b A L M}(x) ; \mu_{r}(x)=\mu_{r P H}(x)+\mu_{r A L M}(x) .
$$

Then the mortality rate after the change point at $x_{0}$ is given

$$
\tilde{\mu}_{r}\left(x_{0}+x\right) \equiv \mu_{r P H}\left(\tilde{W}\left(x_{0}\right)+x\right)+\mu_{r A L M}\left(x_{0}+x\right), x \geq 0 .
$$

Consider the following quotient:

$$
R\left(x, x_{0}\right)=\frac{\mu_{r P H}\left(\tilde{W}\left(x_{0}\right)+x\right)+\mu_{r A L M}\left(x_{0}+x\right)}{\mu_{r P H}\left(x_{0}+x\right)+\mu_{r A L M}\left(x_{0}+x\right)}=\frac{\frac{\mu_{r P H}\left(\tilde{W}\left(x_{0}\right)+x\right)}{\mu_{r A L M}\left(x_{0}+x\right)}+1}{\frac{\mu_{r P H}\left(x_{0}+x\right)}{\mu_{r A L M}\left(x_{0}+x\right)}+1}
$$

Relation $R\left(x, x_{0}\right) \approx 1$ means that the "elasticity part" of an organism dominates. As $\tilde{W}\left(x_{0}\right)>x_{0}$ and we are dealing with increasing mortality rates, the sufficient condition for this is

$$
\mu_{r P H}\left(\tilde{W}\left(x_{0}\right)+x\right)<\mu_{r A L M}\left(x_{0}+x\right),
$$

(the condition $\mu_{r P H}\left(x_{0}+x\right)<<\mu_{r A L M}\left(x_{0}+x\right)$ holds automatically in this case). 
On the other hand, it is obvious that $\tilde{\mu}_{r}\left(x_{0}+x\right) \approx \mu_{r P H}\left(\tilde{W}\left(x_{0}\right)+x\right)$, when

$$
\mu_{r P H}\left(\tilde{W}\left(x_{0}\right)+x\right)>\mu_{r P H}\left(x_{0}+x\right)>>\mu_{r A L M}\left(x_{0}+x\right) \text {. }
$$

In this case:

$$
R\left(x, x_{0}\right) \approx \frac{\mu_{r P H}\left(\tilde{W}\left(x_{0}\right)+x\right)}{\mu_{r P H}\left(x_{0}+x\right)}
$$

and the 'accumulation part' of an organism dominates.

The mentioned above recent findings concerning the mortality plasticity of the fruit flies and other species certainly agree with (37). The human mortality is likely to possess both of these parts. The accumulation (wearing-out) theory contributes to the corresponding age re-calculation rule based on the Sedjakin Principle.

It is a well-known fact in demographic literature that the past (especially in-utero phase, early childhood and adolescence) effects the future mortality patterns of humans (Doblhammer (2003)). On the other hand, there is evidence that with increasing age of the change point $x_{0}$ the effect of the past decreases and humans start to be more susceptible to environmental influence. For instance, the new technology and methods in curing cardio-vascular diseases can have an immediate effect on the mortality of especially the oldest-old group (the saving lives effect). This effect can be perfectly modelled by relations (29)-(31) (see also Vaupel and Yashin (1987)) as the specific form of the $\mathrm{PH}$ model. The recent data on comparative mortality in East and West Germany after unification supports this claim (Scholtz and Maier (2003)). The corresponding mortality curves converge faster for those who were older at $t=1990$. For instance they practically coincide after 1990 for the cohorts born in 1895 (the fast effect due to improvements in economic and social conditions and especially due to the better health care in West Germany). New data should be obtained and new research should be conducted to verify this idea, but it seems rather reasonable from the point of view suggested in this paper.

As it was mentioned while discussing relation (13), environment (life style) can be described by a large number of covariates (parameters). It is reasonable to assume that change points in some parameters are more likely to be described by the plastic model (as in the example of the previous paragraph), whereas change points in other parameters better fit in the framework of the accumulated wear model and the Sedjakin Principle. This agrees with the formal description given by relations (34)-(38). The corresponding generalisation to the multi-parameter case can be easily performed in general but needs a thorough 'experimental' study in the future.

\section{CONCLUDING REMARKS}

We have discussed in this paper certain general approaches to age re-calculation for different mortality regimes and to the choice of the appropriate model for the mortality curve when there is a change point in environment. These models, of course, should be verified on demographic data, but the idea of classifying parameters of environment as leading to the elastic, wear-accumulation, or combined description seems to be rather promising.

It is important to understand that the age-recalculation method defined by the Sedjakin Principle, is a simple rule for using the aggregated information on the past of an object for modeling its future performance. As it was already mentioned, the extensive reliability literature shows that this approach can be reasonable under very mild assumptions, which suggests that it can be used for age re-calculation for organ- 
isms as well. It is worth mentioning, however, that the analogies between stochastic modeling of lifetimes of biological and technical objects should not be understood too literally and the corresponding methods should be used very carefully.

\section{References}

1. Bongaarts J., and Feeney G. (2002). How long do we live? Population and Development Review, 28, 13-29.

2. Block, H.W., Savits T., and Borges W. (1985). Age dependent minimal repair. $J$. Appl. Prob., vol 22, pp.370-386.

3. Cox D. R., and Oakes D. (1984). Analysis of Survival Data, Chapman and Hall, London.

4. Denton F.T., and Spencer B.G. (1999). How old is old? Revising the definition based on life table criteria. Mathematical Population Studies, 7, 147-159.

5. Doblhammer G. (2003). The late life legacy of very early life. Working Paper of the Max Planck Institute for Demographic Research, 2003-030, http://www.demogr.mpg.de/

6. Finkelstein M.S. (1999). Wearing-out components in variable environment. Reliability Engineering and System Safety, 66, N3, 235-242.

7. Finkelstein M. S (1997). The concealed age of distribution functions and the problem of general repair. J. Statist. Plann. Inference, 1997, 65, 315-321.

8. Finkelstein M.S. (2000). Modeling a process of non-ideal repair. In: Recent Advances in Reliability Theory. Limnios N., Nikulin M. (eds). Birkhauser, 41-53.

9. Kolata G.(2003). Low-calorie- diet takes scientists aback. New Yoork Times. 19 September.

10. Liu H., Makish V. (1996) Cutting-tool reliability assessment in variable machine conditions. IEEE Trans. Reliab., 45, 573-581.

11. Mair W., Goymer P., Pletcher S., and Patridge L. (2003). Demography of dietary restriction and death in Drosophila. Science, 301, 1731-1733.

12. Nelson W. (1990). Accelerated Testing. John Wiley \& Sons, New York,

13. Sedjakin N. M. (1966). On one physical principle of reliability theory. Techn. Kibernetika, 3, 80-82 (in Russian).

14. Oeppen J., and Vaupel J.W. (2002). Broken limits to life expectancy. Science, 296, 1029-1031.

15. Shaked, M., and Shantikhumar J. (1993). Stochastic Orders and Their Applications. Academic Press, Boston.

16. Scholtz R., and Mair H. (2003). German unification and the plasticity of mortality at older ages. Working Paper of the Max Planck Institute for Demographic Research, 2003-031, http://www.demogr.mpg.de/

17. Ushakov I. A. and Harrison R.A. (1994). Handbook of Reliability Engineering. John Wiley \& Sons, New Yoork, 1994.

18. Vaupel J.W., and Yashin, A.I. (1987). Repeated resuscitation: how life saving alters life tables. Demography, 4, 123-135

19. Vaupel J.W. (2002). Life expectancy at current rates vs. current conditions. Demographic Research, 7, Article 8 (www.demographic-research.org.).

20. Vaupel J.W., Carey J.R., and Christensen K. (2003). It is never too late. Science 301 (5640), 1679-1681. 